\title{
Molecular mechanisms regulating lifespan and environmental stress responses
}

Saya Kishimoto ${ }^{1,2}$, Masaharu Uno ${ }^{1,2}$ and Eisuke Nishida ${ }^{1,2^{*}}$

\begin{abstract}
Throughout life, organisms are subjected to a variety of environmental perturbations, including temperature, nutrient conditions, and chemical agents. Exposure to external signals induces diverse changes in the physiological conditions of organisms. Genetically identical individuals exhibit highly phenotypic variations, which suggest that environmental variations among individuals can affect their phenotypes in a cumulative and inhomogeneous manner. The organismal phenotypes mediated by environmental conditions involve development, metabolic pathways, fertility, pathological processes, and even lifespan. It is clear that genetic factors influence the lifespan of organisms. Likewise, it is now increasingly recognized that environmental factors also have a large impact on the regulation of aging. Multiple studies have reported on the contribution of epigenetic signatures to the long-lasting phenotypic effects induced by environmental signals. Nevertheless, the mechanism of how environmental stimuli induce epigenetic changes at specific loci, which ultimately elicit phenotypic variations, is still largely unknown. Intriguingly, in some cases, the altered phenotypes associated with epigenetic changes could be stably passed on to the next generations. In this review, we discuss the environmental regulation of organismal viability, that is, longevity and stress resistance, and the relationship between this regulation and epigenetic factors, focusing on studies in the nematode C. elegans.
\end{abstract}

Keywords: Aging, Lifespan extension, Environmental factor, Stress response, Epigenetics, Transgenerational inheritance

\section{Background}

Aging is an inevitable event for most living organisms and is characterized by a progressive decline in physiological function. The aging process is strongly associated with the pathogenesis of many chronic diseases, including cardiovascular disorders, diabetes, cancer, and neurodegenerative diseases. Therefore, understanding the underlying molecular mechanisms of aging could be important for combating age-related diseases. In the 1980s, the isolation of the first long-lived strains of Caenorhabditis elegans established an emerging field of aging research [1]. A number of reports have since identified genetic factors and signaling pathways that are responsible for lifespan regulation [2]. Aging is currently regarded not only as just a passive process of physiological deterioration but also an

\footnotetext{
* Correspondence: eisuke.nishida@riken.jp

${ }^{1}$ RIKEN Center for Biosystems Dynamics Research, 2-2-3 Minatojima Minamimachi, Chuo-ku, Kobe 650-0047, Japan

${ }^{2}$ Department of Cell and Developmental Biology, Graduate School of

Biostudies, Kyoto University, Sakyo-ku, Kyoto 606-8502, Japan
}

actively controlled process that is conserved across species, from yeast to mammals. The well-conserved hallmarks of aging include the accumulation of genomic damages, epigenetic alterations, the loss of proteostasis, and deregulated nutrient sensing [3]. In fact, the aging process is affected by both genetic factors and environmental factors, and these factors are potently correlated with each other [4]. For example, environmental cues such as nutrient intake can interact with chromatin structures and alter transcriptional profiles, which could elicit stable changes in the aging of the organism. In this article, we review the current knowledge of aging research and highlight environmental stress responses that regulate organismal lifespan and stress resistance, with a focus on studies in C. elegans. We also discuss transgenerational effects of ancestral environmental challenges and their underlying molecular mechanisms.

(c) The Author(s). 2018 Open Access This article is distributed under the terms of the Creative Commons Attribution 4.0 International License (http://creativecommons.org/licenses/by/4.0/), which permits unrestricted use, distribution, and reproduction in any medium, provided you give appropriate credit to the original author(s) and the source, provide a link to the Creative Commons license, and indicate if changes were made. The Creative Commons Public Domain Dedication waiver (http://creativecommons.org/publicdomain/zero/1.0/) applies to the data made available in this article, unless otherwise stated. 


\section{Main text}

\section{Insulin/IGF-like signaling pathway in aging}

In 1983, Klass reported the isolation of the first longevity mutants of C. elegans [1], and subsequently, one mutant was named age-1 [5, 6]. The age-1 gene encodes phosphatidylinositol 3-kinase (PI3K), which is a component of the insulin/insulin-like growth factor-1 signaling (IIS) pathway [5, 6]. The IIS pathway plays a pivotal role in the metabolism, growth, and lifespan by sensing nutrient levels. It was first identified as a lifespan-regulating signaling pathway in worms [7]. Many reports have demonstrated that the attenuation of the IIS pathway promotes lifespan extension and stress resistance. For instance, mutations that decrease the activity of daf-2 (the C. elegans homolog of insulin/IGF receptor) more than double the lifespan of the animal [8]. Low IIS activity leads to the activation of the downstream transcription factor DAF-16 (the $C$. elegans homolog of FOXO), and DAF-16 upregulates a wide variety of genes, such as cellular stress response, antimicrobial and metabolic genes, which ultimately exerts pro-longevity effects (Fig. 1) [9-11]. Additionally, heat-shock transcription factor HSF-1 and the antioxidant-regulating transcription factor SKN-1 are also involved in the IIS-mediated lifespan regulation $[12,13]$. The IIS pathway is highly conserved in a wide variety of species, and its suppression extends the lifespan in yeast, flies, and mice [14].

Additionally, TOR (target of rapamycin) signaling is a well-documented signaling pathway that regulates cell survival and growth, and, as in the case of the IIS pathway, the inhibition of TOR activity extends the lifespan of multiple organisms [15-18]. In C. elegans, the prolongevity effect induced by low TOR signaling requires AAK-2 (a catalytic subunit of AMPK) and the transcription factor PHA-4/FOXA, which mediates autophagy (an intracellular proteolytic system crucially involved in

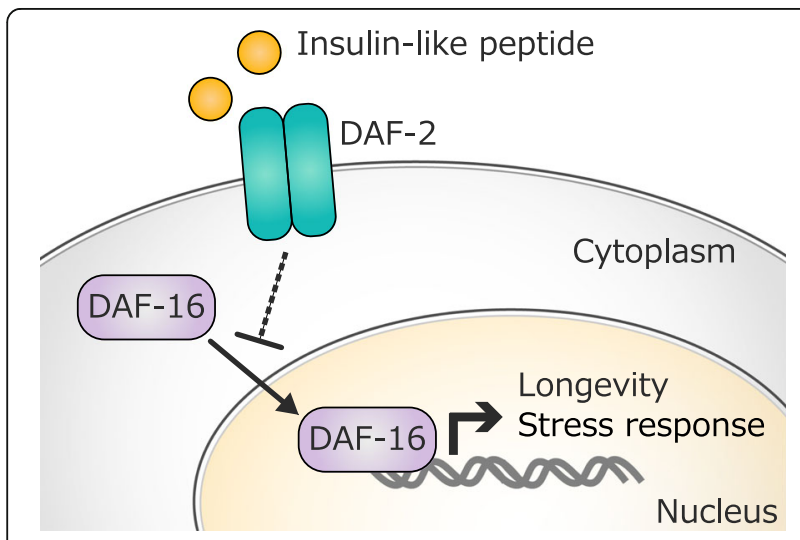

Fig. 1 Schematic diagram of the IIS pathway-mediated longevity in C. elegans. Under reduced IIS, DAF-16 is translocated to the nucleus and activates the transcription of pro-longevity genes lifespan regulation) $[19,20]$. At least in C. elegans, TOR inhibition appears to increase the lifespan independently of DAF-16 [21, 22], suggesting that the TOR pathway may regulate longevity in a manner distinct from the IIS pathway. Recent studies have reported crosstalk between the TOR and IIS pathways for lifespan extension in some contexts $[19,23]$; however, how they interact to modulate longevity in normal physiological conditions remains largely unclear.

\section{Dietary restriction-induced lifespan extension}

Deregulated nutrient sensing is one of the hallmarks of aging [3]; in general, activating nutrient signaling results in the acceleration of aging. It has been consistently reported that dietary restriction (DR, a reduction in food intake without malnutrition) can reduce the nutrient signaling pathway and thereby increase the lifespan and health of many eukaryotes (including yeast, worms, flies, fish, and mice) [24]. DR can also protect against an age-related decline in function and pathologies in mammals. It also reduces the risk factors for cancer, diabetes, and cardiovascular diseases [24]. In addition, it has been reported that DR improves mitochondrial function via sirtuins, including SIRT1 and SIRT3, which contributes to lifespan extension [25]. Many other environmental factors (such as heat stress [26], oxidative stress [27, 28], and pheromones [29]) also induce phenotypic changes, which are relatively stable throughout life and can often prolong the organismal lifespan. Of these, DR is the most effective, well-documented intervention to extend the lifespan in many organisms. Several regimens of DR have been studied [30], including chronic calorie restriction, intermittent fasting, and depletion of specific nutrients such as amino acids. These methods extend the lifespan via distinct mechanisms that partially overlap. It is unlikely that a single pathway mediates the physiological outcomes of DR, as parallel and redundant pathways seem to contribute to the longevity induced by DR [24, 30, 31]. Both the IIS and the TOR signaling pathways sense the nutrient status of organisms. Therefore, the inhibition of these pathways is thought to mimic physiological conditions induced by food shortage. Consistently, genetic analysis has indicated that these pathways are involved in the DR-mediated longevity effects observed in worms and flies [24], although the relevant mechanisms may differ depending on the DR regimen used.

Intermittent fasting (IF) is one of the commonly used dietary restriction methods. In the IF regimen, animals are repeatedly subjected to periods of fasting. In C. elegans, Honjoh et al. demonstrated that IF (every 2 days) dramatically increased the lifespan (by approximately $60 \%$ ) and delays age-related physiological declines [23]. The authors also found that IF-induced longevity is mediated through the TOR signaling pathway. RHEB-1 
(an upstream activator of TOR) induces nuclear translocation of DAF-16, ultimately promoting the transcriptional activation of pro-longevity genes [23]. Another study revealed that, in IF-induced longevity, DAF-16 collaborates with the transcription factor AP-1 (consisting of JUN-1 and FOS-1), and KGB-1 (one of the C. elegans JUNK family members) activates AP-1 in response to fasting [32]. Additionally, SCF E3 ligase complexes are important transcriptional targets of these signaling pathways, and thereby IF induces enhanced protein ubiquitination [32], suggesting that protein homeostasis may contribute to IF-mediated longevity. Similarly, it is well-documented that autophagy, one of the major machineries that regulate protein homeostasis, plays a key role in various longevity pathways, including dietary restriction, in a diverse range of species [33, 34]. Clearance of cell damages by proteolytic systems appears to be important to extend lifespan and delay age-related diseases $[35,36]$.

\section{Epigenetic alterations associated with aging}

Epigenetics is broadly defined as heritable changes in gene function without changes in the DNA coding sequences. The main mechanisms of epigenetic regulation involve DNA methylation, histone modifications, and non-coding RNAs. Epigenetic alterations are relatively stable throughout life and are linked to multiple biological processes, health, and diseases [37, 38]. Intriguingly, some epigenetic signatures have been reported to be biomarkers of aging $[3,39,40]$. For example, increases in histone H3 lysine 4 trimethylation (H3K4me3), H4K16ac, or H4K20me3 and decreases in H3K9me or H3K27me3 are known as age-associated epigenetic marks $[39,40]$. The alteration of these marks is linked to changes in chromatin states around the marks, which may affect gene transcription levels and lead to subsequent biological outcomes. In addition, several studies have demonstrated that genetic manipulations of histone-modifying enzymes can influence the lifespan of multiple organisms. In C. elegans, inhibition of the H3K27me3 demethylase UTX-1 promotes longevity [41, 42]. Deficiency of components of the H3K4me3 methyltransferase complex (composed chiefly of SET-2, ASH-2, and WDR-5) increases lifespan [43]. Consistently, overexpression of the H3K4me3 demethylase RBR-2 extends lifespan, whereas suppression of RBR-2 shortens lifespan [43]. In Drosophila melanogaster, male flies with a deficiency of Lid (the fly ortholog of RBR-2) also show shortened lifespan [44]. However, it is not yet clear how changes in histone modifications regulate the aging process of organisms and whether the effects of histone modification on the regulation of the lifespan are evolutionarily conserved. Further studies are needed to better understand the role of epigenetic alterations in organismal aging. In addition to histone modifications, microRNAs (miRNAs, a class of small non-cording RNAs that post-transcriptionally regulate gene expression) are involved in epigenetic mechanisms, and some miRNAs regulate the lifespan of $C$. elegans under normal physiological conditions [45, 46]. Moreover, several studies have shown that long non-coding RNAs are implicated in longevity $[47,48]$. It has also been reported that other epigenetic alterations, such as DNA methylation and chromatin remodeling, are also associated with aging [49-53].

Epigenetic changes can be modulated by environmental signals. In fact, many metabolites generated by environmental factors, such as ATP and $\mathrm{NAD}^{+}$, often function as cofactors of epigenetic modifiers and substrates [54-57]. This suggests that there is a close relationship between environmental factor-modulated metabolism and epigenetic regulation. Consistent with this concept, epigenetic regulation is relevant to nutrient-sensing pathways, which directly affect metabolism. For instance, it has recently been suggested that MYS-1, the C. elegans homolog of the MYST family histone acetyltransferase Tip60, interacts with TRR-1 (one of PIKK family members) to regulate lifespan and stress resistance through the transcriptional upregulation of DAF-16, possibly mediated by histone acetylation that is catalyzed by MYS-1 [58]. The upregulation of DAF-16/FOXO mediated by the MYST complex was also shown in human cells [58], suggesting that there is an evolutionarily conserved role of histone acetylation. Additionally, a recent study showed that components of the miRNA machinery (including the miRNA-processing enzyme DRSH-1) are required for IF-induced longevity in C. elegans [59]. Moreover, it has been reported that certain miRNAs (miR-228 and miR-71) mediate calorie restriction-induced longevity by interacting with the transcription factors PHA-4 and SKN-1 [60]. These findings suggest that epigenetic mechanisms are associated with the regulation of longevity and stress resistance in response to environmental stimuli. Therefore, epigenetic information may universally integrate environmental inputs throughout life and thus play an important role in the modulation of physiological phenomena, including aging (Fig. 2).

\section{Low-dose environmental stressors and longevity}

Multiple studies have demonstrated that exposure to low-dose environmental stressors elicits beneficial adaptive responses in organisms and increases their survivability, even though higher levels of stress exposure are detrimental [61-63]. The beneficial effects induced by non-lethal exposure to stressors have been called "hormesis" effects. Indeed, stress-induced hormesis effects can contribute to the lifespan extension and increase stress resistance. For instance, animals that undergo mild heat stress can acquire longevity and thermotolerance [64-66]. Such physiologically favorable outcomes of mild stress are 


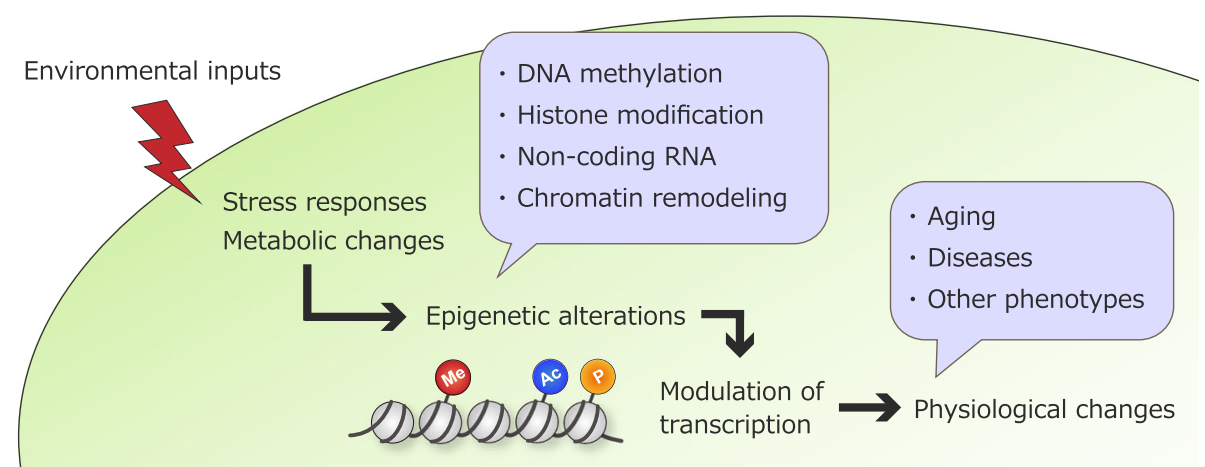

Fig. 2 A possible model of epigenetic regulation in response to environmental factors. The white area represents environmental circumstances, and the green area represents the plausible responses of organisms to environmental stimuli. Epigenetic alterations (such as histone modification and chromatin remodeling) are known as the hallmarks of aging, and these changes are profoundly dictated by environmental stimuli [3, 4, 49]. Me, histone methylation; Ac, histone acetylation; P, histone phosphorylation

considered to largely arise from improvements in the maintenance of cellular homeostasis, such as improved protein quality control. Hormesis effects have been reported across a diverse range of animal species [61]. Recently, it has been shown in C. elegans that exposure to low levels of environmental stressors during the developmental stages increases resistance to oxidative stress and proteotoxicity, suggesting the acquisition of hormesis effects [67]. Intriguingly, the authors found that the hormesis effects acquired in the parental generation could be transmitted to subsequent generations, with offspring showing enhanced stress resistance despite being raised under non-stressed conditions. The transgenerational inheritance continued up to the F3 generation. Moreover, the authors demonstrated that components of the histone H3K4me3 regulatory complex were required for the transgenerational inheritance of the acquired hormesis effects. In the parental generation, the H3K4me3 modifiers functioned in the germline and somehow communicated with DAF-16 and HSF-1 in the somatic tissues to induce and maintain epigenetic alterations. These epigenetic changes appear to be passed on to the next generations and contribute to eliciting hormesis effects for the survival of the offspring (Fig. 3). No direct evidence for the inheritance of stress-induced epigenetic alterations was shown in the study [67]. However, a growing number of studies support the involvement of epigenetic factors in transgenerational inheritance of various physiological changes (discussed below).

\section{Epigenetic regulation on transgenerational inheritance}

Emerging evidence suggests that parental experiences can influence the phenotypes of their offspring in a diverse range of species, including mammals $[68,69]$. This includes "intergenerational" inheritance, which often results from maternal effects or direct exposure of the offspring to environmental cues in utero. However, exposure of only parental males to stimuli has also been shown to induce phenotypic variations in their progeny [70, 71], and these heritable effects could last several generations. These findings suggest that transgenerational inheritance is mediated by invertible and non-genetic mechanisms,
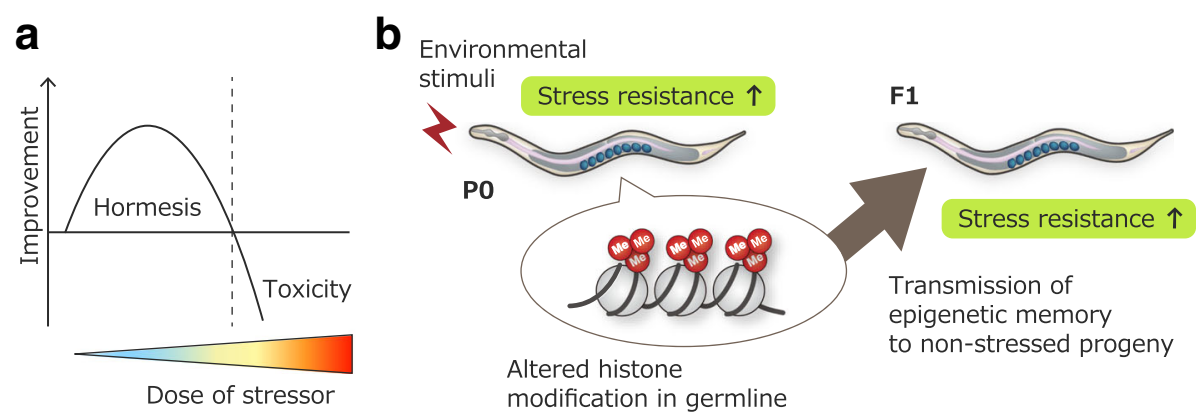

Fig. 3 Transgenerational inheritance of acquired hormesis effects. a Bi-phasic dose-response curve. Low-dose treatment induces enhanced viability (hormesis effects), whereas exposure to high-dose stressors is detrimental. b Schematic model of heritable hormesis effects. Environmental stressors induce epigenetic alterations in the germline, which appear to be transmitted to the next generations and contribute to the viability of the offspring. Me, histone methylation 
presumably epigenetic mechanisms [72-75]. In Drosophila, heat shock-induced heterochromatin disruption was transmitted over multiple generations, presenting as a phenotypic change, but the chromatin state eventually returned to normal [76]. In mice, learning associated with the olfactory system resulted in behavioral and neuroanatomical changes in the descendant generations, which were accompanied by epigenetic alterations involving the olfactory receptor gene [77]. Most examples of transgenerational inheritance are either neutral or harmful to organisms. In some cases, however, beneficial effects induced by parental experiences can be transmitted to the next generations (including the hormesis effect mentioned above [67]). Such heritable phenotypic changes are thought to be an adaptive response that ensures the survival of offspring in harsh environmental conditions.

In the past decade, research in the field has focused on the molecular insights into a non-Mendelian mode of inheritance and has provided some plausible epigenetic mechanisms. In general, reprogramming of the germline removes epigenetic signatures imposed by the environment in parental generations so that the offspring develops properly, according to the appropriate gene regulation. However, epigenetic alterations can sometimes be retained and passed on to the next generation [78]. Recent data in C. elegans provided evidence for the transmission of parental histone modification patterns to embryos [79]. Additionally, many studies have demonstrated that small non-coding RNAs (including miRNA, small interfering RNA (siRNA), and Piwi-interacting RNA (piRNA)) are involved in transgenerational epigenetic inheritance [80, 81]. For example, in C. elegans, starvation in the parental generation alters their small RNA expression profiles, which are maintained and contribute to the longevity of their offspring for multiple generations [82]. Collectively, histone modifications and small RNAs are thought to play a pivotal role in transgenerational inheritance by maintaining ancestral epigenetic memories.

\section{Conclusions}

Organismal lifespan is regulated by both genetic and environmental factors. Genetic mutations (including those in the IIS and TOR pathways) can induce longevity, and environmental stimuli (such as nutrient) also change the aging process. Dietary restriction, one such environmental factor, can effectively extend the lifespan in a diverse range of species. Several factors in the evolutionarily conserved longevity pathways are thought to modulate the epigenetic states of organisms in response to environmental changes and thereby alter their lifespan and stress resistance. In fact, phenotypic changes via epigenetic alterations can continue not only throughout life but also through subsequent generations. Long-lasting epigenetic perturbation seems to be associated with age-related diseases, including cancer and psychiatric disorders, and thus may influence the health and disease state of the offspring [83]. Given the plasticity of epigenetic states, epigenetic modifiers could be potential therapeutic targets. A better understanding of the mechanisms of epigenetic regulation in response to environmental signals may help delay age-related diseases and extend healthy lifespan.

\section{Abbreviations}

DR: Dietary restriction; IF: Intermittent fasting; IIS: Insulin/insulin-like growth factor-1 signaling; miRNA: MicroRNA; TOR: Target of rapamycin

\section{Acknowledgements}

We would like to express our sincere gratitude to Prof. Motoko Yanagita for giving us the opportunity to write this review article and to all lab members and collaborators. We also thank the grants supporting our research.

\section{Funding}

This work was supported in part by grants from Japan Agency for Medical Research and Development (AMED) under grant number 18gm5010001s0501 and 18gm0610017h9905.

\section{Authors' contributions}

SK drafted the manuscript. MU and EN revised it. All authors read and approved the final manuscript.

Ethics approval and consent to participate

Not applicable.

Consent for publications

Not applicable.

\section{Competing interests}

The authors declare that they have no competing interests.

\section{Publisher's Note}

Springer Nature remains neutral with regard to jurisdictional claims in published maps and institutional affiliations.

Received: 1 June 2018 Accepted: 25 July 2018

Published online: 10 December 2018

\section{References}

1. Klass MR. A method for the isolation of longevity mutants in the nematode Caenorhabditis elegans and initial results. Mech Ageing Dev. 1983;22:279-86.

2. Kenyon CJ. The genetics of ageing. Nature. 2010;464:504-12.

3. López-Otín C, Blasco MA, Partridge L, Serrano M, Kroemer G. The hallmarks of aging. Cell. 2013;153:1194-217.

4. Benayoun BA, Pollina EA, Brunet A. Epigenetic regulation of ageing: linking environmental inputs to genomic stability. Nat Rev Mol Cell Biol. 2015;16: 593-610.

5. Friedman DB, Johnson TE. A mutation in the age-1 gene in Caenorhabditis elegans lengthens life and reduces hermaphrodite fertility. Genetics. 1988; 118:75-86.

6. Friedman DB, Johnson TE. Three mutants that extend both mean and maximum life span of the nematode, Caenorhabditis elegans, define the age-1 gene. J Gerontol. 1988;43:B102-9.

7. Kenyon C. The plasticity of aging: insights from long-lived mutants. Cell. 2005;120:449-60.

8. Kenyon C, Chang J, Gensch E, Rudner A, Tabtiang R. A C. elegans mutant that lives twice as long as wild type. Nature. 1993;366:461-4.

9. Lee SS, Kennedy S, Tolonen AC, Ruvkun G. DAF-16 target genes that control C. elegans life-span and metabolism. Science. 2003;300:644-7.

10. Melendez A. Autophagy genes are essential for dauer development and life-span extension in C. elegans. Science. 2003;301:1387-91. 
11. Murphy CT, McCarroll SA, Bargmann Cl, Fraser A, Kamath RS, Ahringer J, et al. Genes that act downstream of DAF-16 to influence the lifespan of Caenorhabditis elegans. Nature. 2003:424:277-84.

12. Hsu A-L, Murphy CT, Kenyon C. Regulation of aging and age-related disease by DAF-16 and heat-shock factor. Science. 2003;300:1142-5.

13. Tullet JMA, Hertweck M, An JH, Baker J, Hwang JY, Liu S, et al. Direct inhibition of the longevity-promoting factor SKN-1 by insulin-like signaling in C. elegans. Cell. 2008;132:1025-38.

14. Tatar M. The endocrine regulation of aging by insulin-like signals. Science. 2003;299:1346-51.

15. Kaeberlein M, Powers RW, Steffen KK, Westman EA, Hu D, Dang N, et al. Regulation of yeast replicative life span by TOR and Sch9 in response to nutrients. Science. 2005;310:1193-6.

16. Kapahi P, Zid BM, Harper T, Koslover D, Sapin V, Benzer S. Regulation of lifespan in Drosophila by modulation of genes in the TOR signaling pathway. Curr Biol. 2004;14:885-90.

17. Jia $\mathrm{K}$, Chen $\mathrm{D}$, Riddle $\mathrm{DL}$. The TOR pathway interacts with the insulin signaling pathway to regulate C. elegans larval development, metabolism and life span. Development. 2004;131:3897-906.

18. Harrison DE, Strong R, Sharp ZD, Nelson JF, Astle CM, Flurkey K, et al. Rapamycin fed late in life extends lifespan in genetically heterogeneous mice. Nature. 2009;460:392-5.

19. Chen D, Li PWL, Goldstein BA, Cai W, Thomas EL, Chen F, et al. Germline signaling mediates the synergistically prolonged longevity produced by double mutations in daf-2 and rsks-1 in C.elegans. Cell Rep. 2013;5:1600-10.

20. Sheaffer $\mathrm{KL}$, Updike DL, Mango SE. The target of rapamycin pathway antagonizes pha-4/FoxA to control development and aging. Curr Biol. 2008; 18:1355-64.

21. Vellai T, Takacs-Vellai K, Zhang Y, Kovacs AL, Orosz L, Müller F. Genetics: influence of TOR kinase on lifespan in C. elegans. Nature. 2003:426:620.

22. Hansen M, Taubert S, Crawford D, Libina N, Lee SJ, Kenyon C. Lifespan extension by conditions that inhibit translation in Caenorhabditis elegans. Aging Cell. 2007;6:95-110.

23. Honjoh S, Yamamoto T, Uno M, Nishida E. Signalling through RHEB-1 mediates intermittent fasting-induced longevity in C. elegans. Nature. 2009; 457:726-30.

24. Fontana L, Partridge L, Longo VD. Extending healthy life span - from yeast to humans. Science. 2010:328:321-6.

25. Ruetenik A, Barrientos A. Dietary restriction, mitochondrial function and aging: from yeast to humans. Biochim Biophys Acta Bioenerg. 2015;1847:1434-47.

26. Xiao R, Zhang B, Dong Y, Gong J, Xu T, Liu J, et al. A genetic program promotes $C$. elegans longevity at cold temperatures via a thermosensitive TRP channel. Cell. 2013;152:806-17.

27. Obata F, Fons CO, Gould AP. Early-life exposure to low-dose oxidants can increase longevity via microbiome remodelling in Drosophila. Nat Commun. 2018;9:975.

28. Schmeisser S, Schmeisser K, Weimer S, Groth M, Priebe S, Fazius E, et al. Mitochondrial hormesis links low-dose arsenite exposure to lifespan extension. Aging Cell. 2013:12:508-17.

29. Ludewig AH, Izrayelit $Y$, Park D, Malik RU, Zimmermann A, Mahanti $P$, et al. Pheromone sensing regulates Caenorhabditis elegans lifespan and stress resistance via the deacetylase SIR-2.1. Proc Natl Acad Sci. 2013;110:5522-7.

30. Mair W, Dillin A. Aging and survival: the genetics of life span extension by dietary restriction. Annu Rev Biochem. 2008;77:727-54.

31. Greer EL, Brunet A. Different dietary restriction regimens extend lifespan by both independent and overlapping genetic pathways in C. elegans. Aging Cell. 2009:8:113-27.

32. Uno M, Honjoh S, Matsuda M, Hoshikawa H, Kishimoto S, Yamamoto T, et al. A fasting-responsive signaling pathway that extends life span in C. elegans. Cell Rep. 2013;3:79-91.

33. Madeo F, Tavernarakis N, Kroemer G. Can autophagy promote longevity? Nat Cell Biol. 2010;12:842-6. https://doi.org/10.1038/ncb0910-842.

34. Nakamura S, Yoshimori T. Autophagy and longevity. Mol Cells. 2018;41:65-72.

35. Rubinsztein DC, Mariño G, Kroemer G. Autophagy and aging. Cell. 2011;146: 682-95.

36. Vilchez D, Saez I, Dillin A. The role of protein clearance mechanisms in organismal ageing and age-related diseases. Nat Commun. 2014;5:1-13.

37. Sidler C, Kovalchuk O, Kovalchuk I. Epigenetic regulation of cellular senescence and aging. Front Genet. 2017;8 SEP:138.

38. Greer EL, Shi Y. Histone methylation: a dynamic mark in health, disease and inheritance. Nat Rev Genet. 2012;13:343-57.
39. Fraga MF, Esteller M. Epigenetics and aging: the targets and the marks. Trends Genet. 2007;23:413-8.

40. Han S, Brunet A. Histone methylation makes its mark on longevity. Trends Cell Biol. 2012;22:42-9.

41. Jin C, Li J, Green CD, Yu X, Tang X, Han D, et al. Histone demethylase UTX-1 regulates $C$. elegans life span by targeting the insulin/IGF-1 signaling pathway. Cell Metab. 2011;14:161-72.

42. Maures TJ, Greer EL, Hauswirth AG, Brunet A. The H3K27 demethylase UTX-1 regulates $C$. elegans lifespan in a germline-independent, insulin-dependent manner. Aging Cell. 2011;10:980-90.

43. Greer EL, Maures TJ, Hauswirth AG, Green EM, Leeman DS, Maro GS, et al. Members of the $\mathrm{H} 3 \mathrm{~K} 4$ trimethylation complex regulate lifespan in a germline-dependent manner in C. elegans. Nature. 2010;466:383-7.

44. Li L, Greer C, Eisenman RN, Secombe J. Essential functions of the histone demethylase lid. PLoS Genet. 2010;6:e1001221.

45. Boehm M, Slack F. A developmental timing microRNA and its target regulate life span in C. elegans. Science. 2005:310:1954-7.

46. Smith-Vikos T, Slack FJ. MicroRNAs and their roles in aging. J Cell Sci. 2012; 125:7-17.

47. Essers PB, Nonnekens J, Goos YJ, Betist MC, Viester MD, Mossink B, et al. A long noncoding RNA on the ribosome is required for lifespan extension. Cell Rep. 2015;10:339-45.

48. Green CD, Huang Y, Dou X, Yang L, Liu Y, Han JDJ. Impact of dietary interventions on noncoding RNA networks and mRNAs encoding chromatin-related factors. Cell Rep. 2017;18:2957-68.

49. Sen P, Shah PP, Nativio R, Berger SL. Epigenetic mechanisms of longevity and aging. Cell. 2016;166:822-39.

50. Lin MJ, Tang LY, Reddy MN, Shen CKJ. DNA methyltransferase gene dDnmt2 and longevity of Drosophila. J Biol Chem. 2005;280:861-4.

51. Matilainen O, Sleiman MSB, Quiros PM, Garcia SMDA, Auwerx J. The chromatin remodeling factor ISW-1 integrates organismal responses against nuclear and mitochondrial stress. Nat Commun. 2017:8:1818.

52. Riedel CG, Dowen RH, Lourenco GF, Kirienko NV, Heimbucher T, West JA, et al. DAF-16 employs the chromatin remodeller SWI/SNF to promote stress resistance and longevity. Nat Cell Biol. 2013;15:491-501.

53. Dang W, Sutphin GL, Dorsey JA, Otte GL, Cao K, Perry RM, et al. Inactivation of yeast Isw2 chromatin remodeling enzyme mimics longevity effect of calorie restriction via induction of genotoxic stress response. Cell Metab. 2014;19:952-66

54. Sharma U, Rando OJ. Metabolic inputs into the epigenome. Cell Metab. 2017;25:544-58

55. Janke R, Dodson AE, Rine J. Metabolism and epigenetics. Annu Rev Cell Dev Biol. 2015:31:473-96.

56. Etchegaray JP, Mostoslavsky R. Interplay between metabolism and epigenetics: a nuclear adaptation to environmental changes. Mol Cell. 2016:62:695-711.

57. van der Knaap JA, Verrijzer CP. Undercover: gene control by metabolites and metabolic enzymes. Genes Dev. 2016:30:2345-69.

58. Ikeda T, Uno M, Honjoh S, Nishida E. The MYST family histone acetyltransferase complex regulates stress resistance and longevity through transcriptional control of DAF-16/FOXO transcription factors. EMBO Rep. 2017;18:e201743907

59. Kogure A, Uno M, Ikeda T, Nishida E. The microRNA machinery regulates fasting-induced changes in gene expression and longevity in Caenorhabditis elegans. J Biol Chem. 2017;292:11300-9.

60. Smith-Vikos T, De Lencastre A, Inukai S, Shlomchik M, Holtrup B, Slack FJ. MicroRNAs mediate dietary-restriction-induced longevity through PHA-4/ FOXA and SKN-1/Nrf transcription factors. Curr Biol. 2014;24:2238-46.

61. Calabrese EJ, Mattson MP. How does hormesis impact biology, toxicology, and medicine? NPJ Aging Mech Dis. 2017;3:13.

62. Yun J, Finkel T. Mitohormesis. Cell Metab. 2014;19:757-66.

63. Gems D, Partridge L. Stress-response hormesis and aging: "that which does not kill us makes us stronger.". Cell Metab. 2008;7:200-3.

64. Kumsta C, Chang JT, Schmalz J, Hansen M. Hormetic heat stress and HSFinduce autophagy to improve survival and proteostasis in C. Elegans. Nat Commun. 2017:8:1-12

65. Hercus MJ, Loeschcke V, Rattan SIS. Lifespan extension of Drosophila melanogaster through hormesis by repeated mild heat stress. Biogerontology. 2003:4:149-56.

66. Lithgow GJ, White TM, Melov S, Johnson TE. Thermotolerance and extended life-span conferred by single-gene mutations and induced by thermal stress. Proc Natl Acad Sci. 1995;92:7540-4. 
67. Kishimoto S, Uno M, Okabe E, Nono M, Nishida E. Environmental stresses induce transgenerationally inheritable survival advantages via germline-tosoma communication in Caenorhabditis elegans. Nat Commun. 2017;8:1-12.

68. Rando OJ. Daddy issues: paternal effects on phenotype. Cell. 2012;151:702-8.

69. Rando OJ, Simmons RA. I'm eating for two: parental dietary effects on offspring metabolism. Cell. 2015;161:93-105.

70. Ng SF, Lin RCY, Laybutt DR, Barres R, Owens JA, Morris MJ. Chronic high-fat diet in fathers programs $\beta$ 2-cell dysfunction in female rat offspring. Nature. 2010;467:963-6.

71. Carone BR, Fauquier L, Habib N, Shea JM, Hart CE, Li R, et al. Paternally induced transgenerational environmental reprogramming of metabolic gene expression in mammals. Cell. 2010;143:1084-96.

72. Szyf M. Nongenetic inheritance and transgenerational epigenetics. Trends Mol Med. 2015;21:134-44.

73. Bohacek J, Mansuy IM. Molecular insights into transgenerational nongenetic inheritance of acquired behaviours. Nat Rev Genet. 2015;16:641-52.

74. Heard E, Martienssen RA. Transgenerational epigenetic inheritance: myths and mechanisms. Cell. 2014;157:95-109.

75. Lim JP, Brunet A. Bridging the transgenerational gap with epigenetic memory. Trends Genet. 2013;29:176-86.

76. Seong KH, Li D, Shimizu H, Nakamura R, Ishii S. Inheritance of stressinduced, ATF-2-dependent epigenetic change. Cell. 2011;145:1049-61.

77. Dias BG, Ressler KJ. Parental olfactory experience influences behavior and neural structure in subsequent generations. Nat Neurosci. 2014;17:89-96.

78. Campos El, Stafford JM, Reinberg D. Epigenetic inheritance: histone bookmarks across generations. Trends Cell Biol. 2014;24:664-74.

79. Gaydos L, Wang W, Strome S. H3K27me and PRC2 transmit a memory of repression across generations and during development. Science. 2014;345: $1515-8$.

80. Rechavi O, Lev I. Principles of transgenerational small RNA inheritance in Caenorhabditis elegans. Curr Biol. 2017;27:R720-30

81. Minkina $\mathrm{O}$, Hunter CP. Intergenerational transmission of gene regulatory information in Caenorhabditis elegans. Trends Genet. 2018;34:54-64.

82. Rechavi O, Houri-Ze'Evi L, Anava S, Goh WSS, Kerk SY, Hannon GJ, et al. Starvation-induced transgenerational inheritance of small RNAs in C elegans. Cell. 2014;158:277-87.

83. Sales VM, Ferguson-Smith AC, Patti ME. Epigenetic mechanisms of transmission of metabolic disease across generations. Cell Metab. 2017;25:559-71.

Ready to submit your research? Choose BMC and benefit from:

- fast, convenient online submission

- thorough peer review by experienced researchers in your field

- rapid publication on acceptance

- support for research data, including large and complex data types

- gold Open Access which fosters wider collaboration and increased citations

- maximum visibility for your research: over $100 \mathrm{M}$ website views per year

At $\mathrm{BMC}$, research is always in progress.

Learn more biomedcentral.com/submissions 